Original Paper http://ajol.info/index.php/ijbcs http://indexmedicus.afro.who.int

\title{
Établissement de quelques valeurs usuelles des paramètres biochimiques chez les chevaux de traction et de sport au Sénégal
}

\author{
A. SOW $^{1 *}$, A. BATHILY ${ }^{1}$, M. KALANDI ${ }^{1}$, D.N. DAO ${ }^{1}$, G.A. OUEDRAOGO ${ }^{2}$ et \\ G.J. SAWADOGO ${ }^{1}$ \\ ${ }^{1}$ École Inter-États des Sciences et Médecine Vétérinaires (EISMV). BP. 5077 Dakar, Fann, Sénégal. \\ ${ }^{2}$ Université Polytechnique de Bobo-Dioulasso, Laboratoire de Recherche et d'Enseignement en Santé et \\ Biotechnologie Animales (LARESBA). BP 01 BP 1091 Bobo-Dioulasso 01, Burkina Faso. \\ *Auteur correspondant; E-mail : wosamada@yahoo.fr; Tél. (+221) 338651008 ; Fax : (+221) 338254283
}

\section{RÉSUME}

L'étude avait pour objectif d'établir quelques valeurs usuelles des paramètres biochimiques chez les chevaux de sport et de traction au Sénégal. Cent quatre-vingt-onze (191) animaux dont 94 chevaux de sport et 97 chevaux de traction âgés de $9,618 \pm 5$, ans et 7,01 $\pm 4,4$ respectivement ont été échantillonnés. Des prélèvements sanguins ont été effectués sur chaque animal pour les analyses biochimiques. Les valeurs usuelles des paramètres biochimiques des chevaux du Sénégal ont été dans le même ordre de grandeur que les valeurs usuelles obtenues chez les chevaux ailleurs, sauf les valeurs de l'ASAT. Les valeurs de l'ASAT et de la créatinine ont été significativement plus élevées $(p<0,05)$ chez de chevaux de race exotique et les chevaux de sport par rapport aux chevaux de race locale et aux chevaux de traction respectivement. De même, des variations significatives $(\mathrm{p}<0,05)$ ont été observées entre les valeurs de l'ASAT, de la créatinine et du magnésium selon les groupes d'âge des animaux. Cette étude a montré que les valeurs des paramètres biochimiques chez les chevaux de sport ne sont pas pleinement extrapolables chez les chevaux de traction au Sénégal. L'établissement des paramètres biochimiques permettra une meilleure prise en charge clinique des chevaux.

(C) 2017 International Formulae Group. All rights reserved.

Mots clés : Chevaux de sport, chevaux de traction, paramètres biochimiques, race locale, race exotique, Sénégal.

\section{Establishment of some reference values of biochemical parameters in working horses and race horses in Senegal}

\section{ABSTRACT}

The objective of the study was to establish some reference values of biochemical parameters in race horses and working horses in Senegal. One hundred and ninety-one (191) animals including 94 race horses and 97 working horses aged of $9.618 \pm 5$ years and $7.01 \pm 4.4$ respectively were sampled. Blood samples were collected from each animal for biochemical analyses. The Student t-test and the analysis of variances (ANOVA) allowed comparing the mean values of the biochemical parameters between the different specific groups. The reference values of the biochemical parameters in horses in Senegal were within the same range as 
the values obtained in the horses elsewhere, except the values of AST. The values of AST and creatinine were significantly higher $(p<0.05)$ in exotic horses and race horses than those in local horses and working horses, respectively. Similarly, significant variations $(p<0.05)$ were observed between the AST, creatinine and magnesium values according to the age groups of the animals. This study showed that the values of biochemical parameters in race horses are not fully applicable in working horses in Senegal. The establishment of biochemical parameters will allow a better healthcare of the horses.

(C) 2017 International Formulae Group. All rights reserved.

Keywords: Race horses, working horses, biochemical parameters, local breed, exotic breed, Senegal.

\section{INTRODUCTION}

Au Sénégal, l'élevage du cheval revêt un intérêt socio-économique certain aussi bien en zone urbaine qu'en milieu rural. Le cheval est utilisé pour le transport des personnes, des marchandises et des ordures ménagères. Dans les campagnes, outre les utilisations déjà citées, le cheval est très sollicité pour les travaux champêtres (Sy, 2004). En effet, le cheval est un auxiliaire agricole indispensable au paysan sénégalais (Bathily et al., 2016). Dans le bassin arachidier, plus de $90 \%$ des exploitations sont équipées d'attelages utilisant la traction équine. Les tractions équines et asines sont utilisées pour les semis et sarclo-binages de l'arachide et des céréales, la récolte de l'arachide et le transport (Havard et al., 2007).

Les courses hippiques sont une tradition de longue date au Sénégal, depuis la période anté coloniale, mais la première compétition officielle fut organisée en 1895 (Ndoye, 1988). Depuis lors, le cheval est bien valorisé dans les sports équestres dont les courses hippiques (Pari Mutuel Urbain), les sauts d'obstacles, la chorégraphie et les randonnées équestres (Tejiozem, 2007).

L'exploitation du cheval est basée essentiellement sur la force musculaire. En raison de leur grande importance, les propriétaires accordent des soins vétérinaires aux chevaux et veillent à leur bien-être. Cependant, peu d'études ont été réalisées sur les paramètres biochimiques des chevaux de traction et de sport au Sénégal. Les cliniciens ne disposent pas suffisamment de données biochimiques pour mieux prendre en charge les chevaux sur le plan sanitaire et alimentaire. Les valeurs usuelles établies chez les chevaux ailleurs ne pourraient pas être pleinement applicables aux chevaux élevés dans les conditions locales du Sénégal en raison des facteurs zootechniques et environnementaux. Or, les analyses biochimiques sanguines représentent un élément important de diagnostic et de pronostic pour le pathologiste en complément de l'examen clinique.

En effet, les analyses biochimiques sanguines sont couramment réalisées en médecine vétérinaire et $\mathrm{s}$ 'intègrent pleinement dans le suivi médico-sportif du cheval de sport et de traction. Les dosages de certains paramètres sanguins sont particulièrement intéressants dans de nombreuses situations en médecine vétérinaire. La réalisation d'un bilan biochimique au repos permet de constituer une référence pour le suivi du cheval et aider ainsi, à détecter de façon précoce les éventuelles inflammations ou dysfonctionnements organiques (Gurgoze et Icen, 2010).

Ainsi, l'objectif de cette étude était de déterminer les paramètres biochimiques des chevaux de traction et de sport et apprécier leurs variations en fonction de l'âge, de la race et du type d'utilisation.

\section{MATÉRIEL ET MÉTHODES}

\section{Site de l'étude}

Les chevaux de traction ont été échantillonnés respectivement dans le bassin arachidier (région de Kaolack) et dans la zone sylvopastorale (région de Saint Louis), deux 
régions agricoles par excellence du Sénégal. Les températures moyennes pendant la période de l'étude variaient entre $35-40{ }^{\circ} \mathrm{C}$. Dans les régions de Kaolack et de SaintLouis, les pluviométries annuelles varient en moyenne entre $850 \mathrm{~mm}$ et $346 \mathrm{~mm}$ respectivement. Pour les deux régions, la force de traction des chevaux est utilisée pour la production agricole et l'exhaure. Les chevaux de sport étudiés provenaient de l'Escadron Monté de la Gendarmerie Nationale de la Caserne Samba Diéry DIALLO de Colobane à Dakar. La Figure 1 donne la localisation des sites de prélèvements.

\section{Échantillonnage}

Une partie de l'échantillon était composée de 97 chevaux mâles entiers de race locale de traction dont l'âge moyen était de 7,66 $\pm 4,6$ ans. Quarante-sept chevaux étaient issus de la région de Kaolack et 50 de la région de Saint-Louis. Ces chevaux étaient nourris avec du fourrage grossier (fanes d'arachide, paille de brousse), de son de riz, de graines de céréales et de l'aliment industriel.

L'autre partie de l'échantillon comportait 94 chevaux de sport âgés en moyenne de 10,20 $\pm 5,3$ ans. Les chevaux de sport étaient composés de 42 animaux de race locale et 52 sujets de races exotiques dont majoritairement la race barbe espagnol. Ces chevaux recevaient une ration alimentaire composée de foins, des aliments concentrés industriels et des compléments alimentaires adaptés aux sportifs.

Au total, l'échantillon comptait 191 sujets dont $139(72,8 \%)$ chevaux de race locale et $52(27,2 \%)$ animaux de races exotiques. Pour une bonne interprétation de l'influence de l'âge sur les paramètres biochimiques, les chevaux de l'étude étaient répartis en 3 groupes d'âge: groupe 1[2-5 ans]; groupe 2]5-10 ans] et groupe 3 ( $>10$ ans). Les moyennes d'âge étaient de $3,93 \pm 1,1$ ans, $7,47 \pm 1,5$ ans et $15,21 \pm 3,3$ ans dans les groupes 1, 2 et 3 respectivement.
Tous les chevaux de cette étude étaient des sujets apparemment en bonne santé (après un examen clinique). Les animaux de moins de 2 ans ou des sujets présentant une affection susceptible de modifier les résultats des prélèvements sanguins ont été exclus de l'étude.

\section{Détermination des paramètres biochimiques}

Des prélèvements sanguins ont été réalisés sur tous les chevaux de l'étude par ponction de la veine jugulaire. Ces prélevements ont été effectués le matin sur tous les animaux sélectionnés. Le sang a été collecté dans des tubes secs stériles sous vide $\left(\right.$ Vacutainer $^{\mathbb{B}}$ ) portant le nom ou numéro de l'animal. Les échantillons sanguins ont été centrifugés ( 3500 tours/min, 15 minutes) et les sérums recueillis ont été conservés à $-20{ }^{\circ} \mathrm{C}$ jusqu'au jour des analyses.

Les analyses biochimiques ont concerné les métabolites (protéines totales, l'albumine, la créatinine et l'urée), les enzymes (aspartate amino-transférase (ASAT) et l'alanine amino-transférase (ALAT)) et les ions (magnésium, phosphore et le calcium). Les analyses ont été réalisées en utilisant des kits commerciaux (Biosystems $^{\circledR}$, S.A., Barcelona, Spain). Les dosages ont été effectués selon le protocole décrit par le fabricant. Les réactions colorimétriques ont été mesurées à l'aide d'un spectrophotomètre (Biosystems-BTS $310^{\circledR}$ ).

\section{Analyses statistiques}

Les données ont été analysées avec le logiciel STATA S.E. 9.2 ${ }^{\circledR}$. Pour chaque paramètre, la moyenne et l'écart type ont été calculés. Le t-test de Student et l'ANOVA ont été utilisés pour comparer les écarts entre les valeurs moyennes des paramètres obtenues (race, utilisation des chevaux, le statut reproducteur et le groupe d'âge). Les différences ont été considérées statistiquement significatives à $\mathrm{p}<0,05$. 


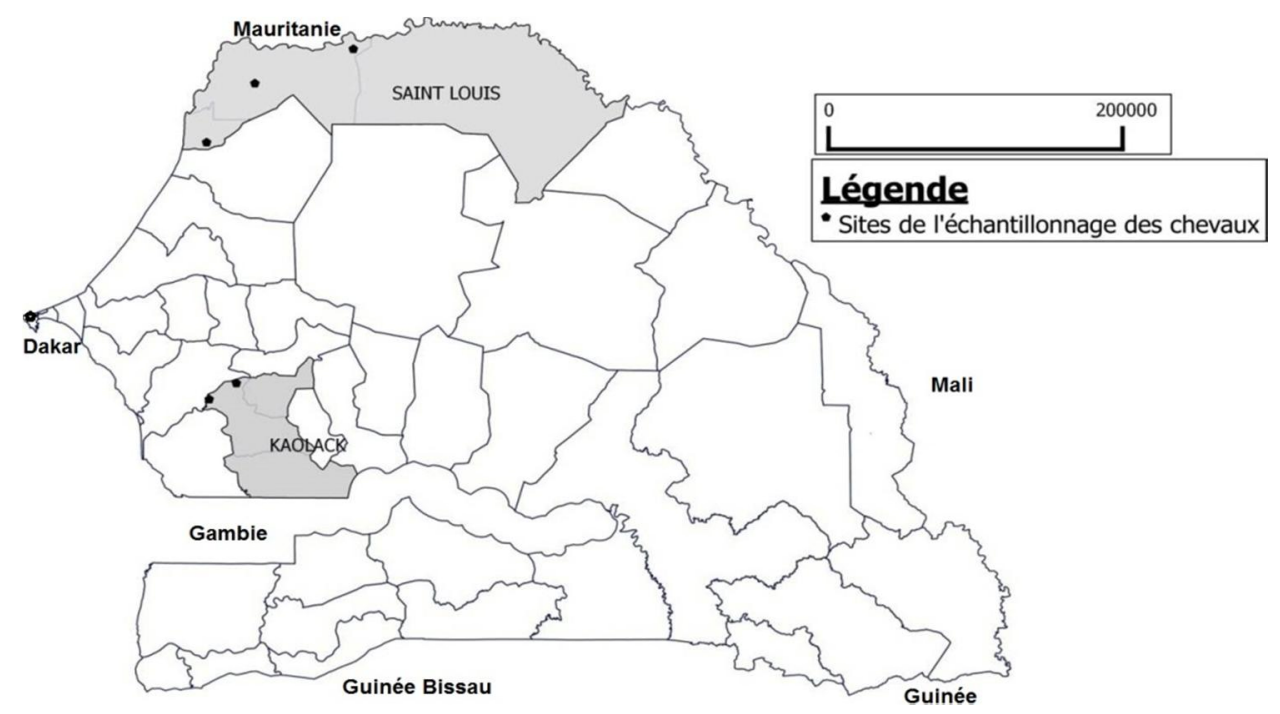

Figure 1 : Localisation des sites d'échantillonnage des chevaux de traction et des chevaux de sport au Sénégal.

\section{RÉULTATS}

\section{Valeurs usuelles moyennes des paramètres} biochimiques

Les valeurs moyennes des paramètres biochimiques pour les chevaux de traction et de sport du Sénégal sont résumées dans le Tableau 1. Les valeurs usuelles moyennes des paramètres biochimiques obtenues sont dans le même ordre de grandeur que celles qui ont été établies dans les études antérieures sur le cheval en général. Toutefois, seules les valeurs de l'ASAT ont été en deçà des valeurs de la littérature ou valeurs de références.

\section{Variations des paramètres biochimiques}

\section{Variation selon la race}

Certaines valeurs des paramètres biochimiques ont varié selon la race des chevaux (Tableau 2). Les valeurs de l'ASAT, des protéines et de l'albumine ont été significativement $(\mathrm{p}<0,05)$ plus élevées chez les sujets de races exotiques que ceux de race locale, tandis que la valeur du magnésium était plus faible chez les races exotiques $(\mathrm{p}<0,05)$.

\section{Variation selon l'utilisation}

Selon que le cheval soit sportif ou utilisé pour la traction, les valeurs de certains paramètres ont varié significativement $(p<0,05)$ (Tableau 3). Ainsi, les chevaux sportifs avaient des valeurs de l'ASAT, de la créatinine, des protéines totales et l'albumine plus élevées que celles des chevaux de traction. Cependant, la valeur de magnésium était plus basse chez les chevaux de sport.

Variation selon le statut reproducteur

Le Tableau 4 montre que les chevaux mâles entiers avaient des valeurs de l'ASAT et de protéines totales plus faibles que chez les chevaux hongres $(p<0,05)$. Tous les autres paramètres avaient à peu près les mêmes valeurs dans les deux groupes.

\section{Variation selon le groupe d'âges}

Pour les trois groupes d'âges, des variations significatives $(p<0,05)$ ont été notées d'un groupe à l'autre (Tableau 5). En effet, la valeur de l'ASAT a été plus élevée chez les animaux des groupes 1 et 2 . Par contre, la valeur des protéines totales était basse chez ces 2 groupes par rapport au groupe 3. Les variations ont également concerné les valeurs de la créatinine et le magnésium. 
Tableau 1 : Valeurs moyennes des paramètres biochimiques des chevaux au Sénégal.

\begin{tabular}{llll}
\hline Paramètres & Total $(\mathbf{n = 1 9 1})$ & Valeurs usuelles & Référence \\
\hline ALAT $(\mathrm{UI} / \mathrm{l})$ & $9,30 \pm 9,1$ & $25,24 \pm 5,8$ & Gul et al., 2007 \\
ASAT $(\mathrm{UI} / \mathrm{l})$ & $145,26 \pm 5,9$ & $229,67 \pm 46,8$ & Gul et al., 2007 \\
Urée $(\mathrm{mmol} / \mathrm{l})$ & $5,11 \pm 1,5$ & $2,2-7,3$ & Niedźwiedź et al., 2013 \\
Créatinine $(\mu \mathrm{mol} / \mathrm{l})$ & $133,94 \pm 32,6$ & $78,3-157,7$ & Tomenendalova et al., 2014 \\
Protéines totales $(\mathrm{g} / \mathrm{l})$ & $66,12 \pm 8,9$ & $69,90 \pm 6,7$ & Bathily et al., 2013 \\
Albumine $(\mathrm{g} / \mathrm{l})$ & $31,43 \pm 7,1$ & $32.45 \pm 8.4$ & Bathily et al., 2013 \\
Magnésium $(\mathrm{mmol} / \mathrm{l})$ & $0,93 \pm 0,3$ & $0,90-1.15$ & Kaneko et al., 2008 \\
Calcium $(\mathrm{mmol} / \mathrm{l})$ & $2,94 \pm 0,8$ & $2,67-3,09$ & Kaneko et al., 2008 \\
\hline
\end{tabular}

Tableau 2 : Variation des valeurs usuelles des paramètres selon la race des chevaux.

\begin{tabular}{lllll}
\hline Paramètres & Race locale $(\mathbf{n}=\mathbf{1 3 9})$ & Race exotiques (n=52) & t-test & p-value \\
\hline ALAT (UI/l) & $8,87 \pm 9,1$ & $10,48 \pm 9,1$ & 1,09 & 0,86 \\
ASAT (UI/l) & $133,68 \pm 56,34$ & $176,22 \pm 50,6$ & 4,77 & $0,000^{* *}$ \\
Urée (mmol/l) & $5,05 \pm 1,6$ & $5,27 \pm 1,3$ & 0,93 & 0,822 \\
Créatinine ( $\mu$ mol/l) & $131,98 \pm 27,2$ & $139,18 \pm 43,8$ & 1,36 & 0,912 \\
Protéines totales $(\mathrm{g} / \mathrm{l})$ & $64,5 \pm 8,68$ & $70,54 \pm 8,12$ & 4,31 & $0,000^{* *}$ \\
Albumine (g/l) & $30,64 \pm 6,02$ & $33,59 \pm 9,3$ & 2,53 & $0,012^{*}$ \\
Magnésium (mmol/l) & $0,96 \pm 0,3$ & $0,85 \pm 0,2$ & 2,27 & $0,012^{*}$ \\
Calcium (mmol/l) & $2,94 \pm 0,6$ & $2,96 \pm 1,2$ & 0,15 & 0,559 \\
\hline * p $<0,05:$ différence significative, $* * \mathrm{p}<0,001:$ différence très significative. & &
\end{tabular}


Tableau 3 : Variation des valeurs usuelles des paramètres biochimiques selon l'utilisation.

\begin{tabular}{lllll}
\hline Paramètres & Traction (n=97) & Sportifs (n=94) & t-test & p-value \\
\hline ALAT (UI/l) & $8,75 \pm 9,9$ & $9,88 \pm 8,2$ & 0,85 & 0,802 \\
ASAT (UI/l) & $135,0 \pm 57,4$ & $155,8 \pm 56,7$ & 2,52 & $0,006^{*}$ \\
Urée (mmol/1) & $5,07 \pm 1,8$ & $5,15 \pm 1,1$ & 0,35 & 0,637 \\
Créatinine $(\mu \mathrm{mol} / \mathrm{l})$ & $128,95 \pm 26,5$ & $139,2 \pm 37,2$ & 2,22 & $0,013^{*}$ \\
Protéines totales $(\mathrm{g} / \mathrm{l})$ & $61,4 \pm 6,0$ & $71,0 \pm 8,9$ & 8,72 & $0,000^{* *}$ \\
Albumine $(\mathrm{g} / \mathrm{l})$ & $30,1 \pm 5,4$ & $32,84 \pm 8,4$ & 2,68 & $0,008^{*}$ \\
Magnésium $(\mathrm{mmol} / \mathrm{l})$ & $1,04 \pm 0,3$ & $0,80 \pm 0,2$ & 6,06 & $0,000^{* *}$ \\
Calcium $(\mathrm{mmol} / \mathrm{l})$ & $2,93 \pm 0,3$ & $2,95 \pm 1,2$ & 0,15 & 0,558
\end{tabular}

Tableau 4 : Variation des paramètres biochimiques selon le statut reproducteur.

\begin{tabular}{lllll}
\hline Paramètres & Mâles entiers (n=180) & Hongres (n=11) & t-test & p-value \\
\hline ALAT (UI/l) & $9,31 \pm 9,1$ & $9,0 \pm 8,7$ & 0,11 & 0,455 \\
ASAT (UI/l) & $145,26 \pm 57,9$ & $186 \pm 50,7$ & 2,46 & $0,014^{*}$ \\
Urée (mmol/l) & $5,11 \pm 1,5$ & $4,77 \pm 0,9$ & 0,76 & 0,222 \\
Créatinine ( $\mu$ mol/l) & $133,94 \pm 32,6$ & $137,16 \pm 46,6$ & 0,34 & 0,632 \\
Protéines totales $(\mathrm{g} / \mathrm{l})$ & $66,12 \pm 8,9$ & $72,1 \pm 9,2$ & 2,31 & $0,011^{*}$ \\
Albumine (g/l) & $31,43 \pm 7,1$ & $34,21 \pm 9,2$ & 1,27 & 0,206 \\
Magnésium (mmol/1) & $0,93 \pm 0,3$ & $0,87 \pm 0,2$ & 0,62 & 0,267 \\
Calcium (mmol/l) & $2,94 \pm 0,8$ & $2,95 \pm 0,8$ & 0,04 & 0,517 \\
\hline * p $<0,05:$ différence significative. & & &
\end{tabular}


Tableau 5 : Variation des valeurs usuelles des paramètres biochimiques selon l'âge.

\begin{tabular}{lllll}
\hline Paramètres & Groupe $\mathbf{1}(\mathbf{n}=\mathbf{6 0})$ & Groupe $\mathbf{2}(\mathbf{n}=\mathbf{6 8})$ & Groupe 3 $(\mathbf{n}=\mathbf{6 0})$ & p-value \\
\hline ALAT $(\mathrm{UI} / \mathrm{l})$ & $9,05 \pm 9,1$ & $8,85 \pm 9,3$ & $10,04 \pm 9,0$ & 0,7322 \\
ASAT $(\mathrm{UI} / \mathrm{l})$ & $153,79 \pm 54,5^{\mathrm{a}}$ & $156,25 \pm 64,3^{\mathrm{a}}$ & $125,28 \pm 48,7^{\mathrm{b}}$ & $0,0032^{*}$ \\
Urée $(\mathrm{mmol} / \mathrm{l})$ & $4,88 \pm 1,7$ & $5,30 \pm 1,6$ & $5,13 \pm 1,2$ & 0,317 \\
Créatinine $(\mu \mathrm{mol} / \mathrm{l})$ & $131,2 \pm 32,9^{\mathrm{a}}$ & $125,51 \pm 27,1^{\mathrm{b}}$ & $145,66 \pm 34,6^{\mathrm{c}}$ & $0,0012^{*}$ \\
Protéines totales $(\mathrm{g} / \mathrm{l})$ & $64,6 \pm 1,7^{\mathrm{a}}$ & $64,64 \pm 8,5^{\mathrm{a}}$ & $69,14 \pm 9,5^{\mathrm{c}}$ & $0,004^{*}$ \\
Albumine $(\mathrm{g} / \mathrm{l})$ & $31,67 \pm 7,7$ & $30,86 \pm 6,4$ & $31,77 \pm 7,3$ & 0,728 \\
Magnésium $(\mathrm{mmol} / \mathrm{l})$ & $0,95 \pm 0,2^{\mathrm{a}}$ & $0,98 \pm 0,3^{\mathrm{b}}$ & $0,85 \pm 0,3^{\mathrm{c}}$ & $0,035^{*}$ \\
Calcium $(\mathrm{mmol} / \mathrm{l})$ & $3,03 \pm 0,3$ & $2,91 \pm 0,6$ & $2,89 \pm 0,8$ & 0,605 \\
\hline
\end{tabular}

\section{DISCUSSION}

De manière générale, toutes les valeurs des paramètres biochimiques obtenues chez les chevaux de traction étaient dans le même ordre de grandeur des valeurs rapportées par des études antérieures (Gul et al., 2007; Kaneko et al., 2008; Bathily et al., 2013; Niedźwiedź et al., 2013; Tomenendalova et al., 2014). L'ASAT a une origine hépatique mais aussi musculaire (Kaneko et al., 2008). La valeur assez faible de l'ASAT obtenue chez les chevaux du Sénégal pourrait être due à la masse corporelle modeste. En effet, le poids vif moyen d'un cheval de race locale au Sénégal est d'environ $270 \mathrm{~kg}$ (Bathily et al., 2016), alors que les races exotiques européennes, américaines ou asiatiques ont un poids moyen d'environ $450 \mathrm{~kg}$ (Tribout, 2013). En effet, l'échantillon comporte $73 \%$ de sujets de race locale. D'ailleurs les chevaux barbe espagnol de l'échantillon avaient des valeurs de l'ASAT nettement supérieures à celles des chevaux locaux.

En plus de l'activité enzymatique de l'ASAT qui a varié significativement selon la race des chevaux, d'autres paramètres comme les protéines totales et le magnésium ont aussi significativement varié. Ces variations pourraient être dues à l'alimentation. En effet, les chevaux locaux constitués essentiellement d'animaux de traction sont nourris à base de fanes d'arachides de sons et des grains de céréales qui sont très riches en magnésium
(Finco, 1997 ; Smith et al., 2006), alors que les chevaux de race exotique constitués de chevaux de sport ont une alimentation bien contrôlée et adaptée aux performances.

Les mêmes tendances de variations des paramètres biochimiques ont été notées chez les chevaux de sport par rapport aux chevaux de traction. Les chevaux de sport sont constitués en majorité $(55,3 \%)$ de sujets de race barbe espagnol. Les valeurs de l'ASAT et de la créatinine étaient significativement plus élevées chez les chevaux sportifs par rapport aux animaux de traction. La variation de l'ASAT pourra s'expliquer par l'activité musculaire plus intense chez les chevaux de sport à cause des entrânements rigoureux qui leur sont imposés. Selon nos résultats, l'activité de l'ASAT était beaucoup plus élevée chez les chevaux hongres que chez les mâles entiers. Les données d'enquête ont montré que ces 8 chevaux hongres étaient des sportifs très expérimentés, ce qui corrobore l'augmentation de l'ASAT avec l'activité musculaire. La concentration de la créatinine évolue avec la masse musculaire (Padalino et al., 2007, Marshall et Bangert, 2005). Or, le poids vif moyen des chevaux de sport au Sénégal atteint $460 \mathrm{~kg}$ (Sow et al., 2016), alors les chevaux de traction constitué de chevaux locaux ne pèsent que $270 \mathrm{~kg}$ en moyenne (Bathily et al., 2016).

L'âge des chevaux a eu un effet significatif sur les valeurs des paramètres 
biochimiques. Les valeurs de l'ASAT du groupe $1(3,93 \pm 1,1$ ans $)$ et $\mathrm{du}$ groupe 2 $(7,47 \pm 1,4$ ans) étaient les mêmes, par contre ces valeurs étaient plus élevées que celles obtenues dans le groupe $3(15,21 \pm 3,3$ ans). Cette variation pourrait être due à l'utilisation plus intense des chevaux de ces deux groupes pour la force de travail pour les chevaux de traction et pour les entraînements intensifs pour les chevaux de sport par rapport aux chevaux du groupe 3 , sujets âgés.

La valeur de la créatinine était plus élevée chez les chevaux du groupe 3 que chez les chevaux du groupe 2 et du groupe 1. En tant que paramètre corrélé à la masse musculaire, ceci peut s'expliquer par le fait que les chevaux du groupe 3 composé d'adultes et vieux chevaux ont une masse corporelle plus importante que les chevaux des deux autres groupes où certains sujets n'ont pas encore achevé leur croissance.

Nos résultats étaient similaires à ceux obtenus par Sow et al. (2014) chez les asins concernant la variation significative du magnésium selon l'âge. En effet, le magnésium est un macroélément essentiel qui est nécessaire pour les réactions dépendantes de l'énergie cellulaire impliquant l'adénosine triphosphate et pour la régulation de la fonction du canal calcique (Stewart, 2011). Par conséquent, il existe une forte corrélation positive entre les ions intracellulaires $\mathrm{du}$ magnésium $\left(\mathrm{Mg}^{2+}\right)$ et les concentrations d'ATP dans les globules rouges de diverses espèces en raison de la présence du complexe $\mathrm{Mg}^{2+}$-ATP au sein des cellules (Miseta et al., 1993). En revanche, Gurgoze et Icen (2010) ont rapporté qu'il n'y a pas d'effet apparent de l'âge sur le niveau de magnésium chez les juments Arabes de race pure.

\section{Conclusion}

La présente étude a permis d'une part, d'établir les valeurs usuelles précieuses de certains paramètres biochimiques chez les chevaux de traction et les chevaux de sport au Sénégal, mais aussi chez les chevaux de race locale et les sujets de race exotique. Ces résultats pourraient aider les vétérinaires à évaluer la condition physique, les performances et le bien-être des chevaux de course et de traction par une bonne prise en charge clinique basée sur l'interprétation des analyses biochimiques. Ces données ont également montré que les paramètres biochimiques des chevaux de sport et des chevaux de race exotique ne sont pas pleinement applicables chez les chevaux de traction et les chevaux de race locale respectivement.

\section{CONFLIT D'INTERETS}

Les auteurs déclarent qu'il n'existe aucun conflit d'intérêt.

\section{CONTRIBUTIONS DES AUTEURS}

$\mathrm{AS}, \mathrm{AB}$ ont rédigé le protocole expérimental, les analyses statistiques et l'interprétation des résultats, $\mathrm{AB}, \mathrm{MK}$ et $\mathrm{DND}$ ont effectué les prélèvements des échantillons et les analyses de laboratoire. AB, MK, GAO, GJS et AS ont réalisé les analyses de laboratoire ainsi que leur interprétation. Tous les auteurs ont contribué à la rédaction du manuscrit.

\section{REMERCIEMENTS}

Nous exprimons aussi notre gratitude au Dr Raphaël TINE, Vétérinaire de l'Escadron Monté du Camp Diéry Diallo de Dakar et les propriétaires des chevaux de traction des régions de Kaolack et de Saint Louis pour leur franche collaboration.

\section{RÉFÉRENCES}

Bathily A, Sow A, Kalandi M, Mouiche MMM, Sawadogogo GJ. 2012. Serum biochemical parameters in racehorses in Senegal. Pak. J. Biochem. Mol. Biol., 45(4): 192-196. http://www.pjbmb.org. pk/images/PJBMBArchive/2012/PJBMB 45_4_Dec_2012/02.pdf

Bathily A, Sow A, Sawadogo GJ. 2016. Paramètres Biochimiques du Cheval de Traction et de Sport au Sénégal: Biochimie du Cheval au Sénégal. Ed UEU: Norderstedt, Germany; 83.

Finco DR. 1997. Kidney function. In Clinical Biochemistry of Domestic Animals. Kaneko JJ, Harvey JW, Bruss ML (eds). Academic Press Inc: San Diego, California, USA; 441-480. 
Gul ST, Ahmad M, Khan A, Hussain I. 2007. Haemato-biochemical observations in apparently healthy equine species. Pakistan Vet. J., 27: 155-158. http://www.pvj.com.pk/pdffiles/27_4/155-158.pdf

Gurgoze SY, Icen H. 2010. The Influence of Age on Clinical Biochemical Parameters in Pure-bred Arabian Mares. J. Eq. Vet. Sci., 30: 569-574. DOI: http://dx.doi.org/10.1016/j.jevs.2010.09. 006

Havard M, Vall E, Njoya A, Fall A. 2007. La traction animale en Afrique de l'Ouest et du Centre. Travaux \& Innovations, 141: 28-32. http://www.pardessuslahaie.net/ journal_trame/ti141_28-32.pdf

Kaneko JJ, Harvey JW, Bruss ML. 2008. Clinical Biochemistry of Domestic Animals (6 ${ }^{\text {th }}$ edn). Academic Press: San Diego, California, USA; 909.

Marshall WJ, Bangert SK. 2005. Clinical Chemistry ( $5^{\text {th }}$ Edn). Elsevier, London, $\mathrm{UK} ; 392$.

Miseta A, Bogner P, Berenyi E, Kellermayer M, Galambos C, Wheatley DN, Cameron IL. 1993. Relationship between cellular ATP, potassium, sodium and magnesium concentrations in mammalian and avian erythrocytes. Biochim. Biophys. Acta., 1175: 133-139. DOI: $10.1016 / 0167-$ 4889(93)90015-H

Ndoye DP. 1988. Le Cheval de Course au Sénégal. Thèse : Méd. Vét. : Dakar, $126 \mathrm{p}$.

Niedzwiedz A, Jaworski Z, Filipowski H, Zawadzki M, Wrzosek M, SluzewskaNiedzwiedz M, Nicpon J. 2013. Serum biochemical reference intervals for the Polish Konik horse (Equus cabalus gmelini Ant.). Vet. Clin. Path., 42(1): 66-69. DOI:10.1111/vcp.12020

Padalino B, Rubino G, Centoducati P, Petazzi F. 2007. Training versus Overtraining: Evaluation of Two Protocols. J. Eq. Vet. Sci. $\quad$ 27: 28-31. DOI: http://dx.doi.org/10.1016/j.jevs.2006.11. 013.

Smith C, Marks A, Lieberman D. 2006. Marks' Basic Medical Biochemistry: A Clinical Approach (2 ${ }^{\text {nd }}$ edn). Lippincott, Williams \& Wilkins: New York, USA; 922.

Sow A, Bathily A, Kalandi M, Tine RS, Sawadogo GJ. 2016. Evolution des paramètres biochimiques chez les chevaux de sport pendant un test d'effort. Int. J. Biol. Chem. Sci., 10(1): 48-57. DOI: http://dx.doi.org/10.4314/ ijbcs.v10i1.5

Sow A, Sidibé I, Kalandi M, Bathily A, Ndiaye NP, Ouédraogo M, Mouiche MMM, Sawadogo GJ. 2014. Biochemical changes induced by natural infection of trypanosomosis in Burkinabese local donkey breeds. Comp. Clin. Pathol., 23: 103-109. DOI: 10.1007/s00580-012-1579-2.

Stewart AJ. 2004. Magnesium Disorders. In Equine Internal Medicine, Reed, Bayly, Sellon (eds, $2^{\text {nd }}$ edn). Saunders Cie: Saint Louis, USA;1365-1379.

Sy I. 2004. Contribution à l'Étude des Lésions Gastro-intestinales d'Origine Parasitaire chez les Chevaux Abattus aux Abattoirs de Dakar. Thèse : Méd. Vét. : Dakar,79p.

Tejiozem GCH. 2007. Utilisation des Produits d'Origine Équine en Thérapeutique Humaine. Thèse : Méd. Vét : Dakar, 97p. Tomenendalova J, Vodicka R, Uhrikova I, Doubek J. 2014. Determination of haematological and biochemical parameters of Przewalski horses (Equus prezwalski) kept by the Prague Zoo. Veterinární Medicína, 59(1): 11-21. http://vri.cz/docs/vetmed/59-1-11.pdf

Tribout PZM. 2013. Étude Morphométrique du Cheval d'Endurance de Race Arabe et Croisé Arabe en Relation avec la Performance. Thèse : Méd. Vét. : Alfort, 94p. 